\title{
The Changing Epidemiology of Urolithiasis in Pediatric Patients
}

Judy N. Chen, Laura Malaga-Dieguez and Howard Trachtman*

Department of Pediatrics, Division of Nephrology, NYU Langone Medical Center, New York, USA

*Corresponding author: Howard Trachtman, NYU Langone Medical Center, CTSI, Room \#110, 227 E 30th Street, New York, USA, Tel: 646-501-2663; Fax: 212-263-4053; E-mail: howard.trachtman@nyumc.org

Rec date: Jan 16, 2014, Acc date: Mar 17, 2014, Pub date: Mar 20, 2014

Copyright: (C) 2014 Chen JN, et al. This is an open-access article distributed under the terms of the Creative Commons Attribution License, which permits unrestricted use, distribution, and reproduction in any medium, provided the original author and source are credited.

\begin{abstract}
The etiology of kidney stones is divided in three broad categories:

(i) Metabolic abnormalities that lead to excessive urinary excretion of solutes those are susceptible to crystallization or reduced excretion of inhibitors of crystallization

(ii) Anatomic defects that cause disturbances in voiding or urinary stasis

(iii) Idiopathic. The laboratory assessment has focused on 24-hour urine collections to measure excretion of calcium, oxalate, citrate, cystine, uric acid, magnesium, and creatinine, selective blood tests, and imaging studies to determine renal structure and function.
\end{abstract}

Keywords: Kidney stones; Urolithiasis; Cardiovascular disease

\section{Introduction}

Urolithiasis has traditionally been considered to be a disease that primarily affects middle age men. The clinical relevance of kidney stones has expanded from a condition associated with acute episodes of pain, need for hospitalization, and loss of work productivity to one that is associated with more significant consequences such as heightened risk of cardiovascular disease. Although there are medical conditions that can cause kidney stones in select populations including children, they are generally rare and have not altered the perception that kidney stones are an adult problem. However, recent findings have challenged this assumption and several reports over the last 5-10 years have documented a rising incidence of urolithiasis in children and adolescents. In this brief review, we will summarize the literature that has addressed the changing epidemiology of urolithiasis in pediatric patients, address the possible causes, and discuss the implications of the trend for general and subspecialty pediatric care.

\section{Overview of Trends in Adults}

A review of the recent literature indicates that the incidence of kidney stone disease is increasing worldwide. For example, in Germany, the incidence of urolithiasis increased from $0.08 \%$ to $0.43 \%$ in adult males, and from 0.04 to 0.29 in adult females between 1979 and 2000. This represents an increase of $170 \%$ [1]. In Japan, the annual incidence rate increased from 54.2 to 68.9 per 100,000 population from 1965 to 1995 based on census data [2]. Finally, the incidence of urolithiasis is also on the rise in the United States, with a greater increase in women. The overall incidence in the U.S. is currently approximately $3 \%$ to $12 \%$ [3]. Studies also show that, in general, incidence is higher among younger patients, which may reflect increased meat protein and salt intake in their diet. However, the development of newer more accurate diagnostic procedures such as sonography and CT has markedly increased the efficacy and accuracy of stone detection and diagnosis and may contribute to the rising incidence of urolithiasis.

\section{Epidemiology of Urolithiasis in Children: Summary of the Literature}

In light of the emerging data about rising incidence of urolithiasis in adults, there has been renewed interest into whether the "stone wave," also applies to children and adolescents. A number of studies have addressed the incidence of urolithiasis in pediatric patients over the last three decades. Methods used to assess incidence rates and patterns of kidney stone disease in children include surveys of hospital admissions, radiological tests, and information on the number of emergency department visits in the pediatric population [4]. One of the first studies to document the changing epidemiology of kidney stones in children was conducted at Schneider Children's Hospital of the North Shore-LIJ Health System. The investigators showed that over a 10-year period, the incidence of urolithiasis in the pediatric referral population increased five times, while the number of patients being evaluated for the disease quadrupled [5]. A review in Japan that focused on kidney disease among children and adolescents from 1965 to 2005 also detected a rising trend, especially in adolescents between the ages of 10 and 19 [6]. Routh et al. utilized the Pediatric Health Information System (PHIS) database to show that kidney stone disease diagnosis increased from 18.4 to 57 per 100,000 patients over the period from 1999 to 2008 [7]. The Rochester Epidemiology Project identified pediatric patients in Olmsted Country, Minnesota diagnosed with kidney stones between 1984 and 2008. In the study, 207 children received a diagnostic code for kidney stone disease, and this was confirmed by review of the medical record in 84 cases. Over the 25 -year period, the incidence rate of urolithiasis increased $4 \%$ per year [8].

The ratio of incidence rates in girls has increased in comparison to that in boys. In a study done in South Carolina, the annual incidence of nephrolithiasis in 2007 was 21.9 and 15.3 per 100,000 for girls and 
boys, respectively. In 1996, the incidence rate were lower and comparable, i.e., for girls 7.7 per 100,000 and for boys 8 per 100,000 [4]. Boys seem to be affected more often in the first decade of their lives, while females were more affected during the second decade. Sas et al. documented that pediatric kidney stone disease was 5.6 times more likely to occur amongst Caucasians than African Americans [9].

Changes in the localization of kidney stones in children and adolescents may be occurring in parallel with the trends in incidence. For instance, in Pakistan and Turkey where pediatric urolithiasis is very common and constitutes a significant cause of end-stage kidney disease, kidney stones were most commonly found in the urinary bladder. However, over a 13-year time frame from 1987 to 2000 , stones are being more commonly detected in the upper urinary tract in children and young adults [10].

In a review of 9,843 urolithiasis cases and 39,047 controls in the PHIS database, a univariate analysis indicated that the stone formers had significantly higher odds of obesity (OR 1.44, 95\% CI 1.27-1.64) and hypertension (OR 2.12, 95\% CI 1.88-2.40) compared to controls. The odds of type I diabetes mellitus was lower among cases compared to controls (OR 0.38, 95\% CI 0.30-0.48). After adjusting for gender, race, insurance type and number of visits, children with urolithiasis still had higher odds of obesity (AOR 1.30, 95\% CI 1.12-1.51) [11]. Other studies suggest that these comorbidities associated with urolithiasis may be affected by age. Children under age 6 who were diagnosed with stone disease were more likely to have hypertension and diabetes mellitus type 1 . In contrast, amongst children age 6 and older, no significant association was identified [12].

\section{Potential Causes}

There are studies linking a number of factors including obesity, various hormones, intake of sweetened beverages, animal protein consumption, and climate change with increased incidence of urolithiasis in the pediatric population.

Approximately $17 \%$ of children ages $2-19$ in the U.S. are overweight with a body mass index (BMI) exceeding the 95th percentile, while $32 \%$ of children are in at least the 85 th percentile [12]. Many metabolic disorders are known to increase concentration of calcium, oxalate, phosphorous, and cystine in the urine. Others reduce the urinary concentration of solutes, such as citrate and magnesium that decrease stone formation [13]. Higher rates of hyperoxaluria, hyperuricosuria, hypercalciuria and hypocitraturia, and renal tubular acidosis have been identified in obese children with higher body fat content [14]. Nonetheless, a direct link between obesity and pediatric stone disease is yet to be established. Body habitus may affect stone formation more than urine chemistry. There is a limited literature which suggests that bone mineralization is linked with urine chemistry leading to stone formation [15]. After puberty, girls experience a sustained increase in adiposity, while boys develop more lean body mass caused by differences in sex hormones. This may help explain the discrepancy in incidence rates between girls and boys.

Diet plays an important role in the development of urolithiasis in the pediatric population. Regular consumption of milk amongst children has declined while sweetened soft drink consumption has risen. Meat protein consumption may also play a role in development of pediatric urolithiasis. The impact of these dietary changes on the epidemiology of urolithiasis in children and adolescents requires further study.
Fakheri and Goldfarb hypothesize that heat-induced sweating caused by higher temperatures from global warming has led to increased kidney stone disease occurrence worldwide. Increased serum osmolality, caused by loss of extracellular fluid via sweating, stimulates secretion of the antidiuretic hormone, vasopressin. Urine volume decreases and salt and calcium concentrations in the urine increase, creating a supersaturated solution. Excess salt precipitates out and crystallizes, leading to the formation of kidney stones. Kidney stone occurrence increases from North to South and from West to East [16]. This factor would account for the rising incidence of kidney stones in pediatric and adult patients.

\section{Clinical Implications}

As the incidence of urolithiasis continues to rise around the world, there will be an increased need for new knowledge and treatment options on the clinician's part. Pediatric practitioners in all settings need to be aware of the possibility of kidney stones in any patient with suggestive symptoms such as abdominal or back pain or abnormalities in the urinalysis. It can no longer be dismissed as solely an adult health problem. The index of suspicion must be high because the symptoms of kidney stones in children may not be as obvious as in adults. Pediatricians will need to be familiar with risk factors associated with the development stones in children and be able to evaluate these patients in a thoughtful and cost effective manner. This will include rational choices of imaging studies to minimize the cumulative exposure of children to X-rays and the appropriate choice of blood and urine tests.

\section{References}

1. Hesse A, Brändle E, Wilbert D, Köhrmann KU, Alken P (2003) Study on the prevalence and incidence of urolithiasis in Germany comparing the years 1979 vs. 2000. Eur Urol 44: 709-713.

2. Yoshida O, Terai A, Ohkawa T, Okada Y (1999) National trend of the incidence of urolithiasis in Japan from 1965 to 1995 . Kidney Int 56: 1899-1904.

3. Persaud AC, Stevenson MD, McMahon DR, Christopher NC (2009) Pediatric urolithiasis: clinical predictors in the emergency department. Pediatrics 124: 888-894.

4. Sas DJ (2011) An update on the changing epidemiology and metabolic risk factors in pediatric kidney stone disease. Clin J Am Soc Nephrol 6: 2062-2068.

5. VanDervoort K, Wiesen J, Frank R, Vento S, Crosby V, et al. (2007) Urolithiasis in pediatric patients: a single center study of incidence, clinical presentation and outcome. J Urol 177: 2300-2305.

6. Yasui T, Iguchi M, Suzuki S, Kohri K (2008) Prevalence and epidemiological characteristics of urolithiasis in Japan: national trends between 1965 and 2005. Urology 71: 209-213.

7. Routh JC, Graham DA, Nelson CP (2010) Epidemiological trends in pediatric urolithiasis at United States freestanding pediatric hospitals. J Urol 184: 1100-1104.

8. Dwyer ME, Krambeck AE, Bergstralh EJ, Milliner DS, Lieske JC, et al. (2012) Temporal trends in incidence of kidney stones among children: a 25-year population based study. J Urol 188: 247-252.

9. Sas DJ, Hulsey TC, Shatat IF, Orak JK (2010) Increasing incidence of kidney stones in children evaluated in the emergency department. J Pediatr 157: 132-137.

10. Clayton DB, Pope JC (2011) The increasing pediatric stone disease problem. Ther Adv Urol 3: 3-12.

11. Kokorowski PJ, Routh JC, Hubert KC, Graham DA, Nelson CP (2012) Association of urolithiasis with systemic conditions among pediatric patients at children's hospitals. J Urol 188: 1618-1622. 
Citation: Chen JN, Dieguez LM, Trachtman H (2014) The Changing Epidemiology of Urolithiasis in Pediatric Patients. J Nephrol Therapeutic S11: S11-006. doi:10.4172/2161-0959.S11-006

Page 3 of 3

12. Matlaga BR, Schaeffer AJ, Novak TE, Trock BJ (2010) Epidemiologic insights into pediatric kidney stone disease. Urol Res 38: 453-457.

13. Bastug F, Düşünsel R (2012) Pediatric urolithiasis: causative factors, diagnosis and medical management. Nat Rev Urol 9: 138-146.

14. Habbig S, Beck BB, Hoppe B (2011) Nephrocalcinosis and urolithiasis in children. Kidney Int 80: 1278-1291.

15. Novak TE, Lakshmanan Y, Trock BJ, Gearhart JP, Matlaga BR (2009) Sex prevalence of pediatric kidney stone disease in the United States: an epidemiologic investigation. Urology 74: 104-107.
16. Fakheri RJ, Goldfarb DS (2011) Ambient temperature as a contributor to kidney stone formation: implications of global warming. Kidney Int 79: 1178-1185. 